Cahiers $d u$ MONDE RUSSE

\section{Cahiers du monde russe}

Russie - Empire russe - Union soviétique et États indépendants

$51 / 4 \mid 2010$

Sciences humaines et sociales en Russie à l'Âge d'argent

\title{
Donald Filtzer, The Hazards of Urban Life in Late Stalinist Russia
}

Jean-Paul Depretto

\section{OpenEdition}

Journals

Édition électronique

URL : https://journals.openedition.org/monderusse/7399

DOI : $10.4000 /$ monderusse. 7399

ISSN : $1777-5388$

Éditeur

Éditions de l'EHESS

\section{Édition imprimée}

Date de publication : 25 novembre 2010

Pagination : 731-737

ISBN : 978-2-7132-2316-7

ISSN : $1252-6576$

Référence électronique

Jean-Paul Depretto, «Donald Filtzer, The Hazards of Urban Life in Late Stalinist Russia », Cahiers du monde russe [En ligne], 51/4 | 2010, mis en ligne le 09 décembre 2011, consulté le 03 septembre 2022. URL : http://journals.openedition.org/monderusse/7399; DOI : https://doi.org/10.4000/ monderusse.7399

Ce document a été généré automatiquement le 3 septembre 2022.

Tous droits réservés 


\title{
Donald Filtzer, The Hazards of Urban Life in Late Stalinist Russia
}

\author{
Jean-Paul Depretto
}

\section{RÉFÉRENCE}

Donald FILTZER, The Hazards of Urban Life in Late Stalinist Russia. Health, Hygiene, and Living Standards, 1949-1953. Cambridge : Cambridge University Press, 2010, 379 p.

1 Ce livre doit être lu comme un complément du volume du même auteur paru en 2002, et dont nous avions rendu compte ici même ${ }^{1}$. Il explore la façon dont les gens (en particulier les ouvriers) vivaient dans les villes de Russie à la fin de la période stalinienne. Une des idées centrales de cette étude est la nécessité d'élargir la compréhension du niveau de vie ouvrier en s'intéressant à la qualité de vie. Les salaires réels seuls donnent une image totalement trompeuse de la vie ouvrière véritable : ainsi, en 1947, ils ont augmenté, mais pour l'écrasante majorité des ouvriers, cette hausse ne fut qu'une illusion: les villes avaient été affectées par la famine et l'approvisionnement restait insuffisant. Suivant l'exemple des historiens de l'industrialisation en Europe de l'Ouest, il faut tenir compte d'aspects de la consommation en URSS négligés jusqu'ici, comme l'accès à des réseaux d'égouts, à une eau sans danger, la possibilité de prendre un bain, etc. ; ces facteurs influençaient l'état de santé de la population et déterminaient le niveau de la mortalité infantile.

2 Donald Filtzer a choisi de centrer son enquête sur ce qu'il appelle l'hinterland industriel de la RSFSR, soit des régions de Russie qui n'ont pas connu de bataille importante ni subi de dommages dus à des opérations militaires pendant la Seconde Guerre mondiale. Cette option présente l'avantage de permettre des comparaisons entre les capitales d'oblast' et les petites villes industrielles qui les entourent, l'auteur soulignant à juste titre qu'une très grande proportion des ouvriers russes vivait dans ces villes. Leur expérience était aussi typique de la vie ouvrière que celles des ouvriers des grandes agglomérations. Or, les historiens ont eu tendance à les négliger. 
3 Trois types principaux de documentation ont été utilisés : 1) les rapports annuels des bureaux locaux de l'Inspection sanitaire d'État, qui dépendait du ministère de la Santé : la plus grande partie de ce que nous savons de l'état du système sanitaire urbain provient de cette source ; 2) les dossiers des ministères de la Santé d'URSS et de RSFSR, complétés par les fonds de la Bibliothèque scientifique centrale de médecine (Moscou); 3) les données de la Direction centrale de la statistique et de la Direction de la statistique de RSFSR sur la démographie et sur la nourriture (enquêtes sur les budgets des ménages, dont il faut encore une fois souligner l'intérêt).

4 Le chapitre I est consacré aux conditions de vie et à leur impact sur la santé. Si la situation peu brillante du logement était connue, Donald Filtzer apporte beaucoup de neuf sur le système sanitaire, sujet peu familier des chercheurs. Seul un petit nombre de villes disposait d'un réseau d'égouts étendu; les gens utilisaient des cabinets extérieurs qui se vidaient dans des fosses d'aisances primitives ou des trous d'écoulement. Les soviets urbains n'étaient pas en mesure de les nettoyer régulièrement, ce qui conduisait à des accumulations d'excréments et d'ordures qui n'étaient enlevées qu'une ou deux fois par an, leur évacuation étant entravée par la pénurie de véhicules, de pétrole, de chevaux et de main-d'œuvre. Pendant la plus grande partie de l'année, les citadins devaient donc circuler dans des rues sales. L'unique exception était Moscou, qui, au début des années 1950, se singularisait par sa propreté - bien qu'une minorité non négligeable de la population n'eût pas encore accès à un réseau d'égouts. Ailleurs, les progrès observés au cours de la période considérée furent lents et inégaux, des projets locaux de construction d'égouts se heurtant au refus du Gosplan d'allouer les crédits nécessaires. Même limités, ces progrès apportèrent cependant une modeste contribution à la baisse de la mortalité infantile due aux infections gastro-intestinales.

5 L'absence d'égouts et d'un système efficace d'évacuation des déchets n'était pas le seul fléau. Il faut y ajouter la difficulté à obtenir l'accès à une eau propre, à la fois pour boire et pour se laver. Les deux problèmes étaient liés, car le manque d'égouts pouvait polluer l'eau. Le deuxième chapitre a précisément pour thème l'approvisionnement des villes en eau et la pollution des rivières. Les grandes villes et beaucoup de petites cités industrielles avaient un approvisionnement en eau centralisé desservant la masse de la population, mais cet indicateur est trompeur car très peu de gens vivaient dans des bâtiments ayant des canalisations internes. L'accès à l'eau était un fardeau domestique majeur : il fallait aller à la pompe dans la rue et ensuite monter les étages avec cette charge. Des investissements et des progrès, limités, furent réalisés dans la distribution de l'eau, mais sans parvenir à répondre à la demande. Même là où les soviets locaux tentaient d'améliorer la situation, ils étaient souvent mis en échec par le refus de Moscou de leur accorder les crédits nécessaires, comme pour les égouts ; les investissements dans ce domaine n'étaient considérés comme importants que dans la mesure où ils affectaient la capacité des usines à remplir le plan.

6 Ajoutons que lorsque les villes possédaient des usines de traitement des eaux d'égout, elles manquaient d'équipement, de pièces détachées et de produits chimiques; de grandes quantités d'eaux sales étaient déversées sans traitement dans les rivières, lacs et étangs. Cette pratique avait des conséquences à court terme sur la santé en créant une menace constante de typhoïde, de dysenterie et autres maladies gastro-intestinales graves, facteurs de mortalité infantile. Plus difficile à mesurer était l'effet à long terme de l'exposition prolongée à la pollution industrielle sur la santé et l'espérance de vie. Dès le début de l'après-guerre, l'Inspection sanitaire d'État avait identifié la pollution 
industrielle comme le plus grand danger : un expert nommé A. Lavrov avait comparé la pollution due aux entreprises et celle imputable aux villes. Ses calculs très grossiers étaient fondés sur un seul indicateur, l'impact de chaque type de pollution sur le contenu de l'eau en oxygène. Lavrov n'avait aucun doute sur la cause de cette dégradation de l'environnement - l'absence de toute planification authentique. De fait, quand ils choisissaient les emplacements d'usines et de cités ouvrières, les ministères n'accordaient aucune attention aux ressources en eau ou aux dommages à prévoir pour les rivières et les lacs à cause des eaux d'égout et des déchets industriels, et ne faisaient aucun effort pour coordonner la localisation des nouvelles entreprises avec les plans d'autres ministères. Malgré le vote de lois, en 1937 et 1947, imposant aux usines l'installation d'équipements antipollution, peu d'entre elles les appliquèrent, le pays ne fabriquant pas le matériel exigé et ne formant pas assez d'ingénieurs et de mécaniciens pour gérer ces systèmes.

7 Lavrov et les inspecteurs sanitaires présents sur le terrain mirent en évidence un aspect essentiel du système soviétique: la croissance économique, à force de polluer l'environnement, engendrait des coûts tels qu'elle se détruisait elle-même. La santé des individus était ruinée et la capacité des ouvriers à créer de la valeur s'en trouvait limitée ; or, c'est cette valeur qui était à l'origine du surplus dont l'élite tirait ses privilèges.

8 Le chapitre III conclut l'étude du système sanitaire par l'examen des bains publics et des moyens utilisés pour empêcher l'éruption et la diffusion du typhus, propagé par les poux. Pour rester propres, les gens comptaient massivement sur les bains russes traditionnels, mais ces bains publics ne pouvaient couvrir qu'une petite fraction des besoins : le plus souvent, il fallait se contenter d'un bain ou deux par mois. La situation était aggravée par une pénurie aiguë de savon. Le but du régime stalinien n'était pas de rendre la vie des citoyens plus supportable, mais de contrôler la maladie. Ne voulant, ou ne pouvant, investir dans les bains publics ou la fabrication de savon, le régime se contentait de mesures strictes pour identifier et isoler ceux qui pouvaient mettre la santé publique en danger : ainsi, les jeunes ouvriers ou les étudiants vivant dans des dortoirs surpeuplés subissaient régulièrement un «traitement sanitaire » visant les porteurs de poux ou les malades du typhus.

Les fonctionnaires des services de santé avaient pour principal souci la sécurité : tant que les gens pouvaient se baigner assez souvent pour contenir la dissémination des poux, les médecins de l'Inspection sanitaire étaient relativement peu concernés. Le danger venait en partie de la politique du régime lui-même: la propagation du typhus était en effet directement liée aux mouvements de population; de ce point de vue, les déplacements de travailleurs forcés et semi-libres présentaient de sérieux risques. Les autorités répondaient par une politique de contrôle rigoureux qui, dans l'ensemble, a réussi à prévenir ou à contenir les éruptions massives de typhus et d'autres maladies contagieuses.

Les dernières années de Stalin marquent le début du déclin de la mortalité infantile et adulte et d'une amélioration de la santé et du bien-être. La plus grande partie de cette réussite était probablement due à une meilleure offre médicale, y compris à l'arrivée des antibiotiques, à des mesures strictes de santé publique et à une amélioration de l'hygiène personnelle. Cette politique fut un succès dans la mesure où elle sut prévenir les épidémies massives. Il demeure que les citoyens continuèrent à subir un environnement urbain souvent sale et la malnutrition. 
11 Le régime alimentaire et la nourriture font l'objet du chapitre IV, où la crise alimentaire de 1947 occupe une place centrale. Cette crise faisait suite à une période de malnutrition massive chronique qui avait affecté de très larges parties de la population. Des millions de personnes étaient mortes de faim pendant la Seconde Guerre mondiale et les survivants étaient très affaiblis. En un sens, cet épisode constitua l'acte final d'une longue crise nutritionnelle qui remontait à la fin des années 1930. Il frappa lourdement les citadins, ouvriers compris, à la différence de la famine de 1932-1933. Mais, dès le début de 1948, la ration alimentaire des familles ouvrières - très bas salaires exclus - augmenta suffisamment pour que leurs vies ne soient plus en danger, malgré le maintien de la malnutrition. Sauf dans les régions de famine du sud de l'Ukraine et de la Moldavie, les paysans étaient mieux placés pour faire face à la crise alimentaire que les ouvriers des villes : le pillage des céréales par l'État avait réduit la place du pain dans la nourriture, mais les paysans purent compenser ce manque grâce aux pommes de terre. Les enquêtes des services statistiques montrent aussi que les paysans consommaient beaucoup plus de lait, ce qui peut expliquer une mortalité infantile plus faible à la campagne. Ces mêmes enquêtes de budgets révèlent aussi l'ampleur de la pauvreté rurale: les familles paysannes n'achetaient presque pas de sous-vêtements ni de chaussures.

12 Les modèles de consommation des familles ouvrières différaient fortement selon les régions: les habitants de Moscou étaient privilégiés, ainsi que (dans une moindre mesure) ceux de Leningrad et de Sverdlovsk, mais même les ouvriers moscovites pâtirent gravement de la crise alimentaire de 1947. Dans les oblasti de Sverdlovsk et de Molotov, l'impact des difficultés put être atténué grâce à la culture de pommes de terre et à la consommation de lait; ce n'était pas vrai de Čeljabinsk et de son oblast', où la mortalité infantile et adulte resta élevée même après 1947. Or Čeljabinsk ne constituait une exception qu'en comparaison de Sverdlovsk et différait peu de la plupart des autres villes et régions : la crise était vraiment générale.

13 Cet épisode apparaît comme la phase finale d'une période de pénurie, commencée avec la guerre et qui se termina fin 1948. Mais les ouvriers et leurs familles continuèrent à recevoir une nourriture inférieure aux normes officielles jusque bien avant dans les années 1950. Ici, il faut se montrer prudent, car la documentation n'est pas claire. Mais des études de la Direction centrale de la statistique indiquent qu'en 1955, la consommation moyenne par tête des familles ouvrières de RSFSR atteignait 2686 calories par jour, soit 200 calories de moins que pour les paysans; ce chiffre se situait en dessous du minimum biologique, tel qu'il était estimé en URSS. Par ailleurs, le régime alimentaire était pauvre en légumes, viande, lait et œufs.

Dans l'URSS d'après-guerre, les possibilités de réduire les activités extérieures au travail étaient limitées: se procurer de la nourriture, de l'eau demandait de grands efforts; il était difficile de maintenir une hygiène personnelle dans des logements dépourvus de toilettes, de bains et d'eau chaude. Dans ces conditions, les gens firent probablement face aux carences alimentaires en réduisant l'intensité au travail : c'est du moins l'hypothèse de Donald Filtzer. La crise alimentaire d'après-guerre a placé les ouvriers dans une situation particulièrement difficile, puisque le besoin physiologique de mettre un frein à l'effort de travail s'est manifesté à un moment où la capacité des travailleurs à s'engager dans une "négociation informelle» avec les managers d'atelier avait notablement diminué. On peut supposer que la nécessité physiologique s'est imposée et que la productivité a diminué : pertes de temps liées aux maladies et accidents, baisse de la production. 

de bien-être d'une population. Son taux s'est accru au début de la guerre, mais a décliné à partir de 1943 (sauf en 1947), tombant à un niveau beaucoup plus bas qu'avant la guerre. Cette amélioration s'est poursuivie jusque dans les années 1970. Le processus, il est vrai, fut inégal selon les régions: les villes industrielles des oblasti de l'Oural et de Sibérie occidentale subirent la situation la plus défavorable. Ce recul connut sa plus grande intensité à Moscou : le taux de mortalité infantile y était bien plus bas que dans toutes les régions de l'hinterland industriel. Malgré ces différences, la tendance sur le long terme était clairement à la baisse et, en 1956, les régions défavorisées commençaient à combler l'écart. Bretagne victorienne et édouardienne ainsi que l'Allemagne wilhelminienne ont vu régresser la mortalité des nourrissons grâce à une confluence de facteurs : amélioration $\mathrm{du}$ système sanitaire et $\mathrm{du}$ logement, baisse du taux de natalité et nourriture moins chère. Cependant, aucun de ces facteurs n'opérait dans l'URSS d'après-guerre où le surpeuplement était aigu, le taux de natalité en hausse, les progrès du système sanitaire urbain très lents, la sous-nutrition systématique et la pénurie de savon et de bains chronique. Comment dès lors expliquer ce succès à première vue paradoxal?

En fait, les mesures de santé publique ont servi de substitut à un processus plus coûteux et plus long de modernisation des infrastructures urbaines. Le niveau d'éducation sanitaire s'est élevé. L'URSS a utilisé des traitements et des savoirs scientifiques occidentaux ; elle a pu soigner la diphtérie et la typhoïde ; elle a commencé à appliquer sous une forme rudimentaire des contrôles épidémiologiques et des techniques d'immunisation de style occidental pour abaisser les taux d'infection et de mortalité de la rougeole parmi les nourrissons et les jeunes enfants. Enfin, diagnostic et hospitalisation sont devenus plus rapides. Ces progrès touchaient une population que ses conditions de vie exposaient constamment à la maladie. Grâce à eux, le régime a réussi à prévenir des éruptions d'épidémies graves et à réduire la mortalité.

De ce point de vue, l'URSS était loin d'être unique : son expérience était comparable à celle de nombreux pays pauvres pendant le deuxième tiers du $\mathrm{xx}^{\mathrm{e}}$ siècle. $\mathrm{Si}$, avant la guerre, le système soviétique de santé publique n'avait pas pu mettre en œuvre les mesures de base qui auraient pu contrecarrer les effets de la pauvreté, après 1945 il n'en allait plus ainsi. Ce phénomène était dû pour une part à la guerre elle-même, qui suscita l'invention de méthodes et de systèmes adéquats pour traiter la forte hausse de la mortalité causée par la détérioration de l'environnement urbain. Ces méthodes furent complétées après la guerre par l'expansion du système de santé, l'usage limité des antibiotiques contre la dysenterie et la pneumonie, et une meilleure conscience publique de l'importance de l'hygiène personnelle. Pour attaquer à la racine la mortalité infantile et la maladie, il aurait fallu améliorer le logement, le système sanitaire urbain, la qualité de l'eau, fournir assez de savon et une bonne alimentation. Le régime ne commença à le faire qu'à la fin de la vie de Stalin : il fallut ainsi attendre les réformes de Hruščev pour voir un progrès plus rapide.

$19 \mathrm{Au}$ total, le livre de Donald Filtzer fait œuvre pionnière dans un domaine très mal connu; il constitue une lecture indispensable pour tous les chercheurs qui s'intéressent à l'histoire sociale soviétique. Son apport le plus neuf consiste à avoir élargi la notion de niveau de vie en y intégrant des composantes qualitatives. Il brasse une quantité impressionnante de documents d'archives et montre tout l'intérêt des fonds du ministère 
de la Santé et des écrits médicaux pour l'histoire sociale. Par une démarche comparative qui n'est pas si fréquente, il replace l'histoire soviétique dans un cadre européen. Saluons aussi l'effort fait par l'auteur pour s'informer sur des questions scientifiques qui ne sont pas forcément familières aux historiens. En outre, on retrouve dans ce volume les qualités pédagogiques bien connues de l'auteur: la langue est toujours claire, les conclusions exposées avec fermeté.

J'ai cependant deux remarques, qui sont autant de questions à Donald Filtzer. Page 17, il est écrit : « ... ce livre a au moins une particularité majeure. C'est un livre sur la façon dont les gens vivaient, mais les gens en sont absents. Les individus, leurs comptes rendus de leurs expériences quotidiennes, ou leurs actions en réponse à leurs expériences sont totalement absents. C'est dans la nature de la documentation». Je n'ignore pas les contraintes imposées par les spécificités des archives soviétiques, où les êtres humains apparaissent plus souvent comme des objets que comme des sujets, mais n'y avait-il aucun moyen de contourner en partie la difficulté, par exemple en utilisant les comptes rendus de réunions organisées par des députés à destination de leurs électeurs, où apparaissent certaines préoccupations quotidiennes de la population? Il me semble en tout cas que la notion d'une histoire sociale sans acteurs est problématique.

21 Ma seconde interrogation porte sur l'emploi de la notion de classe ouvrière: pour l'auteur, cette dernière était "molécularisée " (plutôt qu'atomisée) et "avait perdu le contact avec la plupart des résidus de la conscience de classe» (p. 345-346). Dans ces conditions, pourquoi employer encore le terme de classe? Il me semble que les caractéristiques de la hiérarchie sociale en URSS interdisent cet usage, mais il faudrait en débattre sur le fond ${ }^{2}$.

Bien entendu, ces deux remarques ne changent rien à mon appréciation élogieuse de ce travail pionnier, qui fera date.

\section{NOTES}

1. Soviet Workers and Late Stalinism : Labour and the Restoration of the Stalinist System After World War II, Cambridge : Cambridge University Press, 2002. Cf. Cahiers du Monde russe, 45 (3-4), juilletdécembre 2004, p. 706-709.

2. Cf. mon article «Stratification without Class », Kritika, 8 (2), 2007, p. 375-388. 